\title{
Editorial
}

\section{The Need for Improved Doctoral Program Statistics WILFRED CUDE*}

Across the Western world, there is a clear consensus that no definitive statistics exist on the progress of candidates through doctoral programs. In 1986, the Science and Technology Committee of the Canadian Manufacturers' Association complained that "hard data across all institutions is not available." (CMA, p.1) A year later, a committee surveying information from the major OECD nations, including Scandinavia, France, the United Kingdom and the United States, also complained that "systematic data on non-completion - the extent to which students beginning doctoral training eventually fail to successfully write their thesis - are scanty." (OECD, p.45) Almost simultaneously, the task force of the Economic and Social Research Council in the United Kingdom presented their own report on doctoral submission rates, and commented pointedly on the inadequacy of all available data. Their entire presentation, the task force maintained, had been "seriously hampered by the absence of good data, both inside and outside ESRC." (Winfield, p. 109)

Not content merely to note again a difficulty they recognized as being "remarked on elsewhere," the ESRC task force devoted a full chapter of their report to "The Need for Further Research and Better Data," outlining a spectrum of statistical details requiring immediate attention. Most of these were relevant to at least one of two key areas, each a major statistical objective in itself: "completion times for individual students;" and "withdrawals and failures." (Winfield, p.109) Astonishingly enough for our era of computerized data processing, statistics in the first area are only now appearing from some nations, while statistics in the second area continue to be tentative and highly speculative from every country. Because the United Kingdom is developing the sort of "computerized information system" that distinguishes and enhances contemporary research, the ESRC task force urged all participants to incorporate the relatively minor modifications that would give "ready, cheap access" to the full details of student progress through graduate study. (Winfield, p. 109) Scholars everywhere should support this laudable initiative by insisting on similar measures in their own academic community.

* St. Francis Xavier University 
The present urgency about these statistics stems from the fact that even the existing rudimentary information demonstrates a need for extensive reform of graduate study. Here in Canada, the 1987 annual meeting of the Canadian Association of Graduate Schools expressed concern about both "an alarming drop out rate" among graduate students and "the excessive and increasing time" taken by those successfully completing graduate degrees. Calling the situation a "serious problem," the 39 graduate school deans of CAGS agreed that universities should "tailor degree demands to the length of time during which a fellowship-quality student can reasonably be expected to finish before the fellowship runs out." (University Affairs, p.9) This innocent-sounding objective must necessarily involve a reappraisal of every dimension of graduate study, from administrative regulations right through to methodological considerations. Given the complexity of that enterprise, it should be informed at the outset by the most thorough and reliable data.

The most recent American report on the duration of doctoral programs will serve to drive the point home. Based on 29,991 responses to a survey of the 32,278 persons earning doctorates in the United States during 1987, a statistical sample reaching an almost unprecedented 93 percent of the target group (Coyle, p.iii), this body of data can stand as a model for other related endeavours. Collected by the National Research Council, and placed within the context of results obtained from earlier surveys, these figures demonstrate conclusively that "the time spent in completing a doctoral degree in the United States has been rising steadily for nearly 20 years." One major finding alone will warrant exhaustive analysis. "Time lapse was clearly field-related," the NRC researchers argued: "completion times were shorter in the natural sciences and engineering, about average in the social sciences, and longer in the non-sciences." (Coyle, p.29) However construed, these figures establish that the program is now taking far too long. And they suggest that there may well be other problems with the system.

The discrepancies here are truly startling, particularly within the category of "Median Age at Doctorate," defined as the age at or before which one-half of the successful candidates in a given field received the degree. Ranging from a low of 29 years in Chemistry to a high of 39.8 years in Education, (Coyle, pp. 52-3) these statistics sketch out a profile of an increasingly aging intellectual elite, a cadre of experts just beginning their professional careers when many of their most productive days have already elapsed. While the NRC researchers explore such possible contributing factors as financing, field-switching, employment prospects, citizenship and race, they concede these are not the only variables that "may affect time-to-degree," nor are these "necessarily the most influential." (Coyle, p.32) Carefully excluded from their analysis is the institutional role, a possibility at least recognized by the CAGS, ESRC and 
OECD studies, though not yet extensively explored by anyone. Surely, then, this is an additional reason to press for more and better statistics: once we understand the full parameters of the problem, we can begin to identify the issues most immediately demanding further consideration.

The main area of statistical uncertainty that still remains virtually untouched is the actual drop-out rate among doctoral candidates. Judging from the most reliable estimates currently available, the anxiety of the CAGS graduate deans concerning this subject is well-founded, since the rate does indeed appear unacceptably high. In the United Kingdom, the ESRC researchers have concluded the "wastage rate for Social Science doctorates may be as high as 40\%." (Winfield, p.49) In Canada, von Zur-Muehlen estimates attrition among candidates taking the social sciences as much higher at $50 \%$, a figure he also assigns to those studying the humanities: he only postulates a lower rate among those enrolled in the sciences, engineering and medicine, who withdraw at rates ranging between $35 \%$ and $45 \%$. (von Zur-Muehlen, "Myths," p.20) Commentators are acutely aware that these students have already attained a significant professional qualification, the mastership or its equivalent, before commencing the senior degree. Consequently, attrition at such high levels among people of proven motivation and competence can only create serious doubts about what our universities are doing. This matter has therefore become far too serious to be left any longer in the realm of speculation: estimates like these should either be confirmed or modified, in accordance with the realities of student life.

The awkwardness of proceeding any further with the existing statistics can be illustrated from one of our most comprehensive surveys. In 1978, Dr. Max von Zur-Muehlen of Statistics Canada published a summary of Canadian doctoral statistics over the academic years 1969-70 to 1975-76. Drawing from data provided by each graduate school in the country, this summary gave the total enrolment and number of graduates in every field of study for each year. The result, from one perspective, was quite remarkable: for the first time, scholars could compare relative progress among doctoral candidates in different disciplines, thereby isolating field of study as the most significant variable affecting advancement. The pattern of development now accepted as typical, no matter what the country or decade of investigation, was here indisputably demonstrated. Dividing the total enrolments by the total degrees granted, the Statistics Canada researchers derived an "attainment ratio" for each discipline, a figure that varied widely. On average, the ratio was $20 \%$ in the natural sciences, $7.5 \%$ in the social sciences and $6.7 \%$ in the humanities. (von Zur-Muehlen, "Dilemma," p.79) Placing these ratios in a slightly different relationship, von Zur-Muehlen noted "the humanities and social sciences represent only 33\% of Ph.D. output, although close to $60 \%$ of doctoral enrolment." Obviously, "this 
reflects a longer completion time, and a higher withdrawal rate." (von ZurMuehlen, "Dilemma," p.61) But neither von Zur-Muehlen then, nor any other researcher in any country since, could assign clear percentages to either of these key factors.

This remains a fundamental obscurity in our analysis of graduate programs. Take, for example, the finding that a total enrolment of 5,630 students in English literature yielded a total of 409 doctorates over this period in Canada: we still can only speculate about what those figures mean, both in terms of an actual success rate and the exact longevity of the program. The word "enrolment" almost certainly means "one student enrolled for one academic year," as distinguished from "one student enrolled for the duration of his or her participation:" so how are we to construe our information? Some of those doctorates were granted to people enrolled prior to the period under analysis, so all the enrolments accumulated by these students are not included within the total. Still worse, some of the enrolments in the total must also be credited to people who obtained their doctorates after the period, so neither these extra degrees nor the extra enrolments are included under the respective totals. And worst of all, many of the enrolments must be credited to people who withdrew, but some withdrew during the period, although they enrolled prior to it - and others withdrew after the period, although they enrolled during it. In each case, we have additional enrolments not included under the total. And finally, to further distort an already convoluted statistical situation, we can postulate additional students who enrolled with some of the successful students registered in the total, but who dropped out prior to the period: these enrolments, too, would be relevant yet unrecorded. Our present figures can thus only generate the crudest of estimates concerning the simplest and most important question of all: how many candidates who commence the program actually finish successfully?

We can appreciate the unreliability of the existing attrition estimates by working briefly with a few of the variables involved. Taking again the Canadian figures from English literature, and assuming that enrolment in the discipline did not fluctuate dramatically from year to year, what can we conclude about student success and attrition? During this era, most Canadian universities had a mandatory completion time of six years: so let us further assume that all successful candidates obtained their degrees at or before the lapse of that time. Given a constant rate of enrolment, and a completion time of six years, 409 degrees would account for a maximum of 2454 enrolments: and that would leave some 3176 enrolments unexplained. How, then, to explain them? It is highly unlikely that every candidate who withdrew actually spent up to six years in the program before doing so: thus, if the withdrawing candidates averaged only four years in the program, those 3176 enrolments might represent 
almost 800 unsuccessful candidates, for a real attrition rate of well over $60 \%$. But such argument is quite unacceptable, for several reasons: registration is not constant, the American figures on the longevity of doctoral programs in English would suggest a longer completion time, and nobody can estimate the average length of time withdrawing students spend in their programs. That said, we must grant that objections very much like these can be raised with respect to the accepted estimates: the sharp truth remains that every such exercise is highly suspect, and should be replaced by something better.

The most frustrating aspect of dealing with any attrition estimate is that we all know far better data should be readily available. Cynthia Grover, an official of the Graduate Students' Union at Memorial University of Newfoundland, served our academic community well by tracing the actual success rate of the 98 doctoral candidates enrolled at that institution in the fall of 1982. By the spring of 1989, 59 of those students had graduated, another 26 had withdrawn, and 13 were still enrolled in their programs: hence, the actual attrition rate is only about $30 \%$ so far. (Grover, p.3) It is likely that the 13 students still participating had just enrolled in 1982, and it is therefore conceivable that many of them will actually complete their studies successfully. Should that indeed be the case, then the success rate of these candidates will be higher than the available estimates would suggest. Why don't all our graduate schools undertake similar studies of actual cadres of students, furnishing the full details by field of study? What one graduate student could accomplish in her spare time, an entire institution with computerized data should be able to refine and surpass, drawing from the resources available to faculty and administrative staff. Any Canadian university thus developing hard data on doctoral success rates and attrition would offer a major contribution, not just to the nation, but to the entire academic world.

Scholars everywhere should recognize that a sustained academic reticence about graduate student progress will be open to an increasing and possibly damaging public misconstruction. During this era of economic uncertainty, our universities can no longer risk being perceived as reluctant to scrutinize their own effectiveness. Academic bodies like the ESRC task force have already delicately conceded the impact of "resistance to changes" within the scholarly community, (Winfield, p.115) but other commentators are exercising far less restraint when such resistance continues to impede the precise analysis of what students are obtaining from their institutions. In Halifax recently, one student official responded negatively to news of another tuition increase. "Right now," he said: "we're concerned people aren't getting the quality of education they're paying for. "(Herald, p. A6) Similar thinking has been expressed even more stridently elsewhere. According to Charles J. Sykes, the outspoken author of 
ProfScam, contemporary higher education is "one of society's most outrageous and elaborate frauds," (Sykes, p.4) largely because our academic elite will not countenance serious self-examination. The professorate cannot airily dismiss these criticisms as mindlessly anti-intellectual, since such attacks seem quite plausible in the light of our century-long failure to assess the quality of doctoral study through accurate statistics. The only appropriate response to hostile criticism is good scholarship. It is time we manifested exactly that, in the fundamental statistical profiles of our own professional training.

\section{Des statistiques fiables sur les programmes de doctorat : un besoin pressant}

\section{WILFRED CUDE}

Nous sommes tous d'accord pour déplorer qu'il n'existe pas dans le monde occidental, de statistiques capables d'indiquer avec précision à quel rythme les étudiants inscrits à des programmes de doctorat progressent. En 1986, le Comité consultatif pour les questions scientifiques et technologiques de l'Association des manufacturiers canadiens s'est plaint de "ne pouvoir obtenir de données dures dans tous les établissements que cela regarde" (AMC, p.1). Un an plus tard, un comité chargé d'analyser les renseignements obtenus des principaux pays membres de l'OCDE, la Scandinavie, la France, le RoyaumeUni et les États-Unis, se plaignait de nouveau du "peu de données relatives aux abandons et particulièrement aux causes pour lesquelles des étudiants inscrits à un programme de doctorat ne parvenaient pas à terminer leur thèse"(OCDE, p.45). Presque au même moment, le groupe de travail du conseil de recherches économiques et sociales du Royaume-Uni (ESRC) qui présentait aussi un rapport sur la proportion des thèses déposées soulignait précisément l'insuffisance des données disponibles. L'ensemble de leur étude, affirmait le groupe de travail, "se ressentait grandement du manque de données pertinentes, tant parmi celles du conseil que parmi celles recueillies ailleurs" (Winfield, p.109).

Le groupe de travail ne s'est pas contenté de faire remarquer une fois de plus un inconvénient déjà "observé ailleurs," il a consacré un chapitre entier de son rapport à la "nécessité de poursuivre la recherche et d'obtenir des données plus valables; il suggère, dans ce but, de réunir au plus vite tout un ensemble de renseignements statistiques. Ces renseignements se rapportent à l'un des deux domaines clés suivants, les deux étant en soi des domaines de recherches statistiques fondamentaux : "le temps que les étudiants mettent à terminer leurs études" d'une part et "les abandons et les échecs" d'autre part (Winfield, 
p.109). Chose étonnante à une époque où l'on traite toutes les données par ordinateur, on n'a pu trouver des statistiques relatives à la première question que dans quelques pays, et encore ces statistiques étaient-elles récentes. Quant à la deuxième question, les quelques données qu'on a pu recueillir dans tous les pays sont approximatives, voire de simples suppositions. Comme le RoyaumeUni est en train de mettre en oeuvre un système informatisé de renseignements qui servira à classifier et à promouvoir la recherche dans le monde moderne, le groupe de travail de l'ESRC a demandé que tout le monde introduise dans son système les quelques modifications indispensables à l'obtention de données "fiables et facilement accessibles à un moindre coût". On pourra ainsi disposer de tous les renseignements utiles à l'évaluation du rythme auquel les étudiants progressent dans leurs études (Winfield, p.109). Les universitaires de tous les pays devraient appuyer cette louable initiative en faisant adopter des mesures semblables dans leur université.

La raison pour laquelle il est urgent d'obtenir ces statistiques vient précisément du fait que les données, même rudimentaires, qui ont été recueillies montrent que les études supérieures ont besoin d'être modifiées dans leur organisation. Au Canada, l'Association canadienne des doyens des études avancées s'est dite préoccupée, à sa réunion annuelle de 1987, à la fois par "le nombre effrayant d'abandons" parmi les étudiants de deuxième cycle et par "la lenteur excessive et de plus en plus marquée" de ceux qui viennent à bout de finir leurs études supérieures. Qualifiant cette situation de "sérieuse", les 39 doyens de l'ACDEA se sont entendus sur un principe de base: les universités devraient "revoir l'organisation et les exigences des programmes de sorte que les étudiants titulaires d'une bourse de deuxième cycle puissent normalement remplir les exigences de leur programme d'étude avant la fin de leur bourse" (Affaires Universitaires, p.9). Ce projet pourrait sembler innocent; pourtant il implique nécessairement une évaluation de tous les aspects des programmes d'études supérieures, des procédures administratives jusqu'aux considérations méthodologiques. Une entreprise si complexe doit pouvoir s'appuyer sur des données complètes et fiables.

Le dernier rapport américain en date sur la durée des études de doctorat va nous permettre de bien faire comprendre notre point de vue. Ce rapport s'appuie sur un sondage mené auprès des 32278 personnes qui ont obtenu leur doctorat aux États-Unis en 1987. Sur ce nombre, 29991 ont répondu, soit $93 \%$ du groupe ciblé, ce qui ne s'était peut-être jamais vu (Coyle, p.iii). Ces données pourront donc servir de modèle à toutes les autres recherches sur ce sujet. Réunis par le Conseil national de recherches et placés dans la perspective des résultats obtenus à l'occasion de sondages antérieurs, les chiffres montrent 
indubitablement “que le temps pris pour terminer un doctorat n'a pas cessé de s'allonger aux États-Unis ces vingt dernières années." Parmi les conclusions tirées de ce rapport l'une d'elle justifierait à elle seule une analyse exhaustive: "la durée dépendrait de toute évidence du champ d'étude." Les chercheurs du CNR ajoutent même que "la durée est plus courte en sciences naturelles et en ingénierie, moyenne en sciences sociales, et plus longue dans les domaines non scientifiques" (Coyle, p.79). De quelque façon qu'on les interprète, ces chiffres montrent que les programmes d'études supérieures durent bien trop longtemps. Ils suggèrent de plus que certains problèmes pourraient venir de l'organisation de ces études.

Les écarts sont en effet tout à fait surprenants, particulièrement dans la catégorie intitulée "âge moyen des lauréats". Cet âge moyen est calculé à partir de l'âge auquel ou avant lequel la moitié des candidats ont obtenu leur doctorat dans une matière donnée. Les résultats montrent un minimum de 29 ans en chimie et un maximum de 39,8 ans en éducation (Coyle, p.52-53), ce qui dénote un vieillissement constant de l'élite intellectuelle et laisse présager l'émergence d'un groupe d'experts qui commenceront seulement leur carrière au moment où leurs années de production maximale sont déjà passées. Les chercheurs du CNR s'interrogent sur les causes possibles d'une telle situation: le coût par exemple, la décision de changer de spécialisation, les perspectives d'emploi, mais aussi certaines préférences reliées à une appartenance ethnique ou raciale. Ils admettent volontiers qu'il pourrait y avoir d'autres facteurs ayant pu contribuer à prolonger les études et que ceux qu'ils ont retenus ne sont pas nécessairement les plus déterminants (Coyle, p.37). Toutefois, on remarque qu'ils ont eu soin d'exclure de leur analyse le rôle joué par les institutions, rôle dont l'ACDEA, l'ESRC et l'OCDE avaient pourtant au moins reconnu l'existence probable, même si aucune de leurs recherches n'avait fourni de renseignements approfondis à ce sujet. Voilà donc une raison supplémentaire de réclamer d'autres recherches statistiques et des données plus fiables: une fois que nous connaîtrons tous les paramètres du problème, nous pourrons considérer quels aspects demandent à être traités en priorité.

Il y a un domaine où les statistiques n'apportent pour ainsi dire aucune réponse: c'est celui du taux réel d'abandon chez les candidats au doctorat. $\mathrm{Si}$ l'on en juge par les estimations les plus fiables dont on dispose actuellement, les craintes de l'ACDEA sur cette question sont des plus fondées car ce taux semble en effet extraordinairement élevé et tout à fait inacceptable. Au Royaume-Uni, les chercheurs de l'ESRC ont avancé "le chiffre de $40 \%$ d'abandons parmi les étudiants en sciences sociales" (Winfield, p.49). Von ZurMuehlen estime qu'au Canada les abandons parmi les étudiants en sciences 
sociales pourraient même atteindre $50 \%$, chiffre qu'il applique également aux lettres et aux sciences humaines: il n'y a qu'en science, en ingénierie et en médecine qu'il estime le taux inférieur et le situe entre 35 et 45 pour cent (Von Zur-Muehlen, "Myths", p.20). Or tous ces chercheurs sont parfaitement conscients du fait que les étudiants dont il s'agit avaient déjà tous atteint un niveau de spécialisation appréciable (la maîtrise ou l'équivalent) avant d'entreprendre leur doctorat. En conséquence on peut dire qu'un taux d'abandon si élevé parmi des gens dont on ne peut douter de la motivation ni de la compétence oblige à se poser de sérieuses questions sur les universités. Il est clair que ceci est bien trop grave pour qu'on en reste à de pures spéculations: de telles estimations doivent maintenant être confirmées ou corrigées, selon ce qui se passe réellement.

Nous pouvons illustrer les difficultés auxquelles les chercheurs se heurtent lorsqu'ils utilisent les statistiques actuelles pour faire avancer nos connaissances grâce à l'une des études les plus complètes que nous possédions. En 1978, monsieur Max Von Zur-Muehlen de Statistique Canada a publié un compte rendu des statistiques disponibles alors sur les doctorats faits au Canada entre 1969 et 1976. Il se fondait sur les données qu'il avait reçues de tous les établissements d'études supérieures du pays et présentait le nombre total d'inscriptions et le nombre d'étudiants par matière pour chacune de ces années universitaires. Le résultat a été d'un certain point de vue tout à fait remarquable: pour la première fois, les chercheurs pouvaient comparer le temps pris par les étudiants des diverses disciplines, ce qui a permis de voir que la durée variait d'abord en fonction de la matière. Le modèle de progression que l'on considère maintenant comme typique, dans quelque pays que ce soit ou sur quelque époque que porte l'enquête, se trouvait ici indiscutablement démontré. En divisant le nombre d'étudiants inscrits par le nombre de diplômes décernés, les chercheurs de Statistique Canada ont trouvé pour chaque matière un taux de réussite dont le chiffre pouvait varier grandement. On obtenait en moyenne un taux de $20 \%$ en sciences naturelles, de 7,5\% en sciences sociales et de 6,7\% en lettres et sciences humaines (Von Zur-Muehlen, "Dilemma", p.79). En interprétant les chiffres dans une perspective légèrement différente, Von ZurMuehlen a remarqué de plus que "les lettres, les sciences humaines et les sciences sociales ne représentaient que $33 \%$ des doctorats décernés tandis qu'à l'inscription elles représentaient $60 \%$ des étudiants." De toute évidence "les étudiants mettent plus de temps à finir dans ces domaines, et il y a plus d'abandons" (Von Zur-Muehlen,"Dilemma", p.61). Toutefois, ni Von ZurMuehlen à cette époque, ni aucun chercheur depuis n'a pu présenter de pourcentages exacts sur cette question pourtant essentielle.

La signification des chiffres reste d'ailleurs obscure dans notre analyse aussi. 
Prenons par exemple les chiffres suivants: des 5630 étudiants inscrits en doctorat de littérature anglaise à cette époque, 409 ont reçu leur diplôme. Ce que l'on peut tirer de ces chiffres ne saurait être autre chose que de pures spéculations tant sur le taux réel de réussite que sur la durée du programme. Le terme "inscription" signifie en effet très certainement "étudiant inscrit au cours d'une année universitaire", ce qui ne veut pas dire "étudiant inscrit pendant tout le temps qu'il suivait le programme." Comment pourrons-nous donc interpréter ces chiffres? Il y a eu des doctorats décernés pendant la période étudiée à des étudiants inscrits bien avant; les inscriptions antérieures de ces étudiants ne font pas partie du total. Mais il y a pire: certaines inscriptions ont pu mener, après la période étudiée, à l'obtention d'un doctorat; les inscriptions postérieures et les diplômes décernés n'apparaissent pas non plus dans les statistiques. Enfin, un grand nombre des inscriptions retenues viennent sans doute de personnes qui ont abandonné leurs études; toutefois certaines de celles qui ont abandonné pendant cette période s'étaient inscrites avant tandis que d'autres, inscrites pendant cette période, ont abandonné après. Dans tous les cas, il faut rajouter des inscriptions au total que nous possédons. Finalement, pour bien montrer que nos connaissances statistiques sont encore pires que nous le pensions, il faudrait ajouter les étudiants qui se sont inscrits en même temps que certains lauréats inclus au total, mais ont abandonné avant la période analysée: ces inscriptions aussi auraient un sens; et pourtant elles n'ont jamais été considérées. Les chiffres dont on dispose actuellement ne peuvent donc produire que de vagues estimations lorsqu'on cherche à répondre à la question, la plus simple et la plus importante de toutes: combien, sur tel montant d'étudiants s'inscrivant à un programme de doctorat, y aura-t-il de lauréats?

Nous pouvons apprécier le manque de pertinence des estimations actuelles relatives aux abandons en considérant brièvement quelques-unes des variables qui touchent à cette question. Reprenons d'abord les chiffres donnés pour la littérature anglaise au Canada. Il est probable que le nombre d'inscriptions n'a pas beaucoup changé d'une année sur l'autre dans cette matière. Quelles conclusions pouvons nous donc tirer des chiffres que nous possedons par rapport au taux de réussite et au taux d'abandon? Pendant cette période, la plupart des universités canadiennes exigeaient que l'étudiant termine ses études en six ans maximum: nous pouvons en déduire que les lauréats ont dû obtenir leur diplôme au bout de six ans ou avant la fin de cette période. En partant d'un nombre d'inscriptions constant et de l'obtention du diplôme dans la limite de temps prévue, 409 doctorats signifieraient au plus 2454 inscriptions, ce qui ne permettrait pas d'expliquer les 3176 inscriptions restantes. À quoi cela tient-il? Il est peu probable que tous les candidats ayant abandonné aient été inscrits pendant les six ans du programme avant de se retirer; alors, si on calcule une 
moyenne de quatre ans pour ceux qui ont abandonné, ces 3176 inscriptions pourraient signifier presque 800 échecs et un taux réel d'abandon nettement supérieur à $60 \%$ ! Un tel résultat paraît toutefois parfaitement inacceptable pour plusieurs raisons: le nombre d'inscriptions n'est pas constant, les chiffres américains sur la durée des programmes de doctorat d'anglais montrent qu'ils prennent plus de six ans, et personne ne peut estimer au bout de combien de temps ceux qui ne terminent pas abandonnent. Ceci dit, force nous est d'admettre qu'on peut soulever des objections très semblables à celles-ci à propos des estimations que nous avons retenues: la vérité, c'est que toutes ces spéculations sont profondément suspectes et demandent à être remplacées par autre chose.

Ce qu'il y a de plus frustrant quand on se penche sur le taux d'abandon tel qu'estimé, c'est que l'on sait pertinemment qu'il serait facile d'obtenir des chiffres fiables. Cynthia Grover, représentante officielle du syndicat des étudiants de deuxième cycle à la Memorial University of Newfoundland (université de Terre-Neuve), a rendu un grand service au monde universitaire en présentant le taux réel de réussite des 98 étudiants inscrits à un programme de doctorat dans cette institution en automne 1982. En 1989, au printemps, 59 de ces étudiants avaient obtenu leur diplôme, 26 avaient abandonné leurs études et 13 étudiaient toujours. Cela fait un taux réel d'abandon de seulement $30 \%$ à ce moment (Grover, p.3). Il est probable que les treize étudiants qui n'avaient pas fini à ce moment s'étaient inscrits pour la première fois en 1982 et l'on peut supposer que la plupart d'entre eux auront terminé leurs études peu après. Si c'était le cas, le taux de réussite des étudiants en doctorat serait bien supérieur aux estimations réalisées jusqu'alors. Pourquoi les établissements d'enseignement supérieur n'entreprennent-ils pas une enquête semblable sur leurs étudiants, enquête qui fournirait tous les renseignements importants par champ d'étude? Sans doute, ce qu'une étudiante a accompli sur ses heures de loisir, un établissement entier disposant d'un système informatisé peut le faire, et même le faire mieux, avec l'aide des professeurs et du personnel administratif. L'université canadienne qui entreprendrait de réunir des données de ce type sur les taux de réussite et d'abandon au niveau du doctorat apporterait une contribution essentielle, non seulement à son pays mais aussi au monde universitaire international.

Les universitaires doivent comprendre qu'en rechignant à entreprendre des recherches sur la façcon dont les étudiants progressent dans leurs études, ils risquent de transmettre une idée fausse au public, idée qui pourrait faire beaucoup de tort aux universités. À une époque d'incertitude économique comme celle que nous traversons actuellement, les universités ne peuvent plus se montrer réticentes à évaluer leur propre efficacité. Même des organismes 
constitués d'universitaires tel le groupe de travail de l'ESRC ont bien voulu admettre implicitement l'importance du rôle que jouait dans cette question la "résistance au changement" qui caractérise l'ensemble de la communauté universitaire (Winfield, p.115); d'autres critiques se montrent encore plus sévères, surtout lorsque cette résistance continue à empêcher d'étudier avec précision ce que les étudiants tirent de leurs établissements. Récemment, à Halifax, un représentant étudiant a refusé catégoriquement une nouvelle augmentation des frais de scolarité. "Actuellement," a-t-il affirmé, "nous ne sommes pas sûrs d'en avoir pour notre argent" (Herald, p.A6). Cette idée a été exprimée avec encore plus de virulence ailleurs. Selon Charles J. Sykes, l'auteur de ProfScam (la duperie des profs) dont nous connaissons le francparler, l'enseignement supérieur est actuellement "l'une des fraudes les plus scandaleuses et les mieux organisées qui soient" (Sykes, p.4), et ce surtout parce que l'élite universitaire ne veut pas entendre parler de faire son autocritique. Le corps professoral ne peut plus rejeter ces commentaires avec désinvolture en disant simplement qu'elles sont stupides et ne visent qu'à dénigrer les intellectuels! Celles-ci paraissent en effet de plus en plus fondées lorsqu'on considère que, depuis un siècle, l'université se montre incapable d'évaluer la qualité des études de doctorat qu'elle propose au moyen de statistiques précises. La seule façon valable de réagir à des critiques hostiles, c'est de faire preuve d'honnêteté intellectuelle, en menant des recherches sérieuses capables de définir au moyen de statistiques quels sont les fondements de notre formation professionnelle.

\section{REFERENCES}

Coyle, Susan L. (1989). Summary report 1987: Doctorate recipients from United States universities. Washington, D.C.: National Academy Press.

Editorial staff. (1990, June 28). Value for the money. Halifax Chronicle-Herald.

Graduate deans unhappy about time taken to finish degrees. (1988, March). University Affairs.

Grover, Cynthia. (1989) How many PhDs? Memorial University Graduate Students' Union New'sletter, 2 (3).

Organization for Economic Co-operation and Development (1987). Post-graduate education in the 1980s. Paris: OECD Publication Service.

Science \& Technology Committee (1986). University post graduate training: Is the pipeline too long? Montreal: Canadian Manufacturers' Association.

Sykes, Charles J. (1988). ProfScam: Professors and the demise of higher education. Washington, D.C.: Regenery Gateway.

von Zur-Muehlen, Max (1987). Myths and realities: The fallacy of faculty shortages in the next decade. The Canadian Journal of Higher Education, 17 (1): 13-25.

von Zur-Muehlen, Max (1978). The Ph.D. dilemma in Canada revisited. The Canadian Journal of Higher Education. 8(1): 49-92.

Winfield, Graham et. al (1987). The social science PhD: The ESRC inquiry of submission rates. London: Economic and Social Research Council. 